\title{
A MEDIDA DA INOVAÇÃO FARMACÊUTICA E OS PEDIDOS DE PATENTE: o caso da doença de Chagas ${ }^{1} / /$ Marcos Vinício Chein Feres ${ }^{2}$, Alan Rossi Silva ${ }^{3}$, Anderson Resende Morais ${ }^{4}$ e Andressa Mendes de Souza ${ }^{5}$
}

\author{
Palavras-chave \\ pesquisa empírica em direito / inovação \\ farmacêutica / pedidos de patente / doenças \\ negligenciadas / doença de Chagas
}

\section{HIIIIIIIIIIIIIIIIIIIIIIIIIIIIIIIIII}

\section{Sumário}

\section{Introdução}

2 Estratégias teórico-metodológicas

2.1 Medida: confiabilidade e validade

2.2 Inovação Farmacêutica

2.3 Doença de Chagas

2.4 Coleta de Dados

2.5 Limitações

3 Depósitos de pedidos de patente relacionados à doença de Chagas

4 Os depósitos de pedidos de patente e a medida da inovação farmacêutica

5 Conclusão

6 Referências

\begin{abstract}
Resumo
O presente estudo visa a investigar se o número de depósitos de pedido de patente, relacionados à determinada doença, constitui-se como medida confiável e válida para se aferir os níveis de inovação farmacêutica na realidade brasileira. Para se alcançar esse objetivo, foi realizada, na base de dados oficial do INPI, uma coleta dos depósitos de pedidos de patente relacionados à doença de Chagas. Como substrato teórico-metodológico, utilizaram-se as regras de inferência, de Epstein e King, bem como o conceito de inovação farmacêutica, de Marcia Angell. A partir dos dados analisados, ao final, foi possível inferir, em primeiro lugar, que o número de depósitos de pedidos de patente, relacionados à determinada doença, pode ser considerado uma medida confiável da inovação farmacêutica, caso a coleta dos depósitos, em base oficial do INPI, seja norteada por regras precisas, transparentes e replicáveis. E, em segundo lugar, foi possível inferir que, apesar de confiável, por não ser considerada uma medida visual, imparcial e eficientemente válida, o número de depósitos de pedidos de patente, relacionados à determinada doença, não pode ser considerado uma medida válida para se aferir os níveis de inovação farmacêutica na realidade brasileira.
\end{abstract}

\footnotetext{
1 Este trabalho possui financiamento da Fundação de Amparo à Pesquisa de Minas Gerais (FAPEMIG), do Conselho Nacional de Desenvolvimento Científico e Tecnológico (CNPq) e da Coordenação de Aperfeiçoamento de Pessoal de Nível Superior (CAPES).

2 Doutor em Direito, Professor Associado da Universidade Federal de Juiz de Fora (UFJF) e Professor do Corpo Permanente do Programa de Pós-Graduação Estrito Senso em Direito e Inovação da Faculdade de Direito da UFJF. Bolsista de Produtividade PQ-2 do CNPQ. E-mail:<mvchein@gmail.com>.

3 Mestrando em Direito e Inovação na Universidade Federal de Juiz de Fora (UFJF). Bolsista do Programa Demanda Social, Auxílio Financeiro, pela Coordenação de Aperfeiçoamento de Pessoal de Nível Superior (CAPES). E-mail: <alanrossi100@gmail.com>.

4 Graduado em Direito pela Universidade Federal de Juiz de Fora (UFJF). Bolsista de Apoio Técnico - BAT, da Fundação de Amparo à Pesquisa do Estado de Minas Gerais (FAPEMIG). E-mail: <resendemorais@vol.com.br>.

5 Graduanda em Direito pela Universidade Federal de Juiz de Fora (UFJF). Bolsista do Programa Institucional de Bolsas de Iniciação Científica e Tecnológica (PROBIC), da Fundação de Amparo à Pesquisa do Estado de Minas Gerais (FAPEMIG). E-mail:<souza.andressamendes@gmail.com>.
} 


\section{THE MEASURE OF PHARMACEUTICAL INNOVATION AND PATENT REQUESTS: the case of Chagas disease // Marcos Vinício Chein Feres, Alan Rossi Silva, Anderson Resende Morais and Andressa Mendes de Souza}

\section{Keywords}

empirical legal research / pharmaceutical innovation / patent requests / neglected diseases / Chagas disease

HIIIIIIIIIIIIIIIIIIIIIIIIIIIIIIIII

\begin{abstract}
This paper aims to investigate whether the number of patent requests related to a specific disease can be considered a reliable and valid measure of pharmaceutical innovation in Brazil. To accomplish this goal, data collection was carried out in the INPI database, concerning patent requests directly related to Chagas disease. The theoretical and the methodological frameworks, applied to interpret the data collection, were elaborated from the rules of inference, developed by Epstein and King, and from the concept of pharmaceutical innovation, formulated by Marcia Angell. Finally, it was possible to infer that the number of patent requests related to a specific disease could be considered a reliable measure of pharmaceutical innovation, if the data mining in the INPI database is guided by accurate, transparent and replicable rules. Moreover, it was also possible to infer that, despite being reliable, the number of patent requests related to a specific disease is not a valid measure, as it can not be considered a facially valid, unbiased, efficient measurement procedure of pharmaceutical innovation in Brazil.
\end{abstract}




\section{Introdução}

Atualmente, tanto no ambiente acadêmico quanto nos movimentos sociais, há uma grande controvérsia em relação ao diagnóstico do desempenho da inovação farmacêutica em geral ${ }^{6}$ (Kesselheim, Wang \& Avorn, 2013). Como se pode observar no estudo realizado por Kesselheim, Wang e Avorn (2013), enquanto alguns estudiosos afirmam que o ritmo da inovação farmacêutica vem diminuindo, outros afirmam exatamente o contrário.

Nesse estudo, Kesselheim, Wang e Avorn (2013) realizaram uma revisão sistemática da literatura científica sobre essa controvérsia, com o intuito de desenvolver uma taxonomia própria dos procedimentos utilizados para se medir a inovação farmacêutica em cada um dos estudos analisados. Para tanto, os autores coletaram 42 estudos, que foram divididos em quatro categorias principais de procedimentos de medida utilizados: contagem de novas drogas aprovadas, avaliação do valor terapêutico, resultados econômicos e patentes concedidas (Kesselheim, Wang \& Avorn, 2013). Como resultado, os autores concluíram que a controvérsia existente sobre o desempenho da inovação farmacêutica, manifestada pela variação dos resultados dos diferentes estudos analisados, está diretamente relacionada à própria definição de inovação farmacêutica e ao procedimento de medida utilizado em cada investigação (Kesselheim, Wang \& Avorn, 2013).

Também nesta direção, Boldrin e Levine (2013) destacam a relevância dessa discussão ao demonstrarem que a utilização do número de patentes concedidas como procedimento de medida provoca uma inflação injustificada dos índices de inovação. Segundo os autores, apesar do grande crescimento do número de patentes concedidas ao longo das últimas décadas, na economia dos Estados Unidos, não se observa uma aceleração correspondente do progresso tecnológico, nem dos níveis de investimento em pesquisa e desenvolvimento (P\&D) (Boldrin \& Levine, 2013). Em

6 Nesse sentido, em relação ao meio acadêmico, verifique, por exemplo, os estudos realizados por Pedrique et al. (2013), por Feres e Silva (2017), por Boldrin e Levine (2013) e por Gold et al. (2010). Em relação às controvérsias apresentadas pelos movimentos sociais, veja, por exemplo, o relatório dos Médicos Sem Fronteiras (Médecins Sans Frontières, 2016) e da Drugs for Neglected Diseases initiative (DNDi, 2015). outras palavras, o número de patentes concedidas, segundo os autores, não seria uma medida adequada para se verificar os níveis de inovação tecnológica (Boldrin \& Levine, 2013).

Além das formas citadas, o número de pedidos de patente depositados (Garcia \& Gonçalves, 2016) (Pomin, 2015) (Silva \& Luz Junior, 2016), a quantidade de artigos publicados relacionados a determinado assunto (Antunes, 2003) (Silva \& Luz Junior, 2016) e o número de pesquisadores relacionados à determinada área (Antunes, 2003) são outros exemplos de medidas utilizadas pela literatura científica da área, para se mensurar a inovação farmacêutica amplamente.

Com isso, pode-se observar a pluralidade de entendimentos sobre o melhor procedimento de medida a ser utilizado e a importância dessa discussão metodológica para se elaborar um diagnóstico confiável do desempenho da inovação farmacêutica no Brasil. Nesse sentido, não obstante todas as possibilidades mencionadas acima, a presente investigação visa a verificar, especificamente, se o número de depósitos de pedidos de patente, relacionados à determinada doença, constitui-se como medida confiável e válida para se aferir os níveis de inovação farmacêutica na realidade brasileira.

Como hipótese levantada inicialmente a essa questão, considerando os critérios de confiabilidade e validade das medidas elaboradas por Epstein e King (2013) e o conceito de inovação farmacêutica elaborado por Angell (2007), afirma-se que o número de pedidos de patente depositados, relacionados à determinada doença, apesar de poder ser considerado confiável - caso a coleta dos depósitos, em base oficial do Instituto Nacional da Propriedade Industrial (INPI, 2017), seja norteada por regras precisas, transparentes e replicáveis -, não se constitui como um procedimento de medida válido para se mensurar os níveis de inovação farmacêutica no Brasil.

Para se investigar o questionamento supracitado, na próxima seção serão expostas as estratégias teórico-metodológicas utilizadas, onde os conceitos utilizados serão esclarecidos e o procedimento de coleta de dados será descrito minuciosamente. Em seguida, os 
dados coletados na base de dados do INPI (2017) serão expostos e analisados descritivamente. Depois, a partir da análise dos dados conhecidos, realizar-se-ão as inferências descritivas, que sustentarão as conclusões do presente estudo. Por fim, na última parte do trabalho, serão expostas as conclusões acerca do conteúdo desenvolvido e serão elencados alguns possíveis desdobramentos da presente investigação.

\section{Estratégias teórico-metodológicas}

Para alcançar os objetivos propostos inicialmente, é necessário esclarecer, em primeiro lugar, o caráter empírico do presente estudo. Embora a comunidade jurídica possa ter atribuído um significado particularmente restrito à palavra "empírico" - associada, geralmente, a técnicas e análises estatísticas ou a dados quantitativos -, segundo Epstein e King (2013) "a palavra empírico denota evidência sobre o mundo baseada em observação ou experiência" (p. 11). Essa evidência, segundo os autores, pode ser tanto quantitativa (numérica) quanto qualitativa (não-numérica), não havendo hierarquia entre elas. Assim, de acordo com essa concepção, o que caracteriza a presente pesquisa empírica é, justamente, o fato de ela ser baseada em dados da realidade, independentemente de ser baseada em números ou em letras ${ }^{7}$ (Epstein \& King, 2013).

Nesse caso específico, para alcançar os fins propostos pela presente investigação, a estratégia metodológica utilizada será composta apenas por elementos quantitativos (análise numérica dos pedidos de patentes), a partir dos quais será possível desenvolver inferências descritivas ${ }^{8}$ (Epstein \& King, 2013).

\subsection{Medida: confiabilidade e validade}

Como o objetivo da presente investigação é o de avaliar a confiabilidade e a validade dos números de depósitos de pedido de patente como medidas de inovação farmacêutica, faz-se necessário, nesta seção, delimitar três conceitos fundamentais: "medida",

7 Sobre essa distinção, entre pesquisa quantitativa (números) e pesquisa qualitativa (letras), no âmbito das ciências sociais, são também importantes as contribuições de Pires (2008).

8 De acordo com Epstein e King (2013), inferência descritiva é o processo de se utilizar fatos conhecidos para se aprender sobre fatos desconhecidos. "confiabilidade" e "validade".

Segundo Epstein e King (2013), o ato de medida consiste em "comparar um objeto de estudo (por exemplo, um evento, sujeito ou processo do mundo real) com algum standard (padrão), como aqueles existentes para quantidades, capacidades ou categorias" (pag. 101). Apesar de considerarem a medida insuficiente para se compreender os fenômenos e as complexidades do mundo real em sua totalidade, os autores consideram que ela desempenha um papel fundamental na pesquisa empírica, uma vez que, para entender a realidade, sempre será necessário algum nível de abstração e, consequentemente, redução das particularidades existentes no mundo (Epstein \& King, 2013).

Nesse sentido, dada a importância dos métodos de medida para a pesquisa empírica, de acordo com Epstein e King (2013), uma medida deve ser avaliada em duas dimensões: quanto à sua confiabilidade e quanto à sua validade. Para os autores, confiabilidade "é a extensão à qual se pode replicar uma medida, reproduzindo o mesmo valor (indiferente de ser este o valor correto ou não) no mesmo padrão para o mesmo tópico a um mesmo tempo"' (Epstein \& King, 2013, p. 105). Isso significa dizer que, em uma pesquisa empírica, para uma medida ser considerada confiável, ela precisa produzir o mesmo resultado repetidamente, não importando quem está realizando a medida (Epstein \& King, 2013).

Para que uma medida possa ser confiável e produzir resultados independentemente do agente medidor, é fundamental que o julgamento e a interpretação humana sejam reduzidos ao máximo (Epstein \& King, 2013). E, nos casos em que for imprescindível, regras precisas sobre os procedimentos adotados devem ser elaboradas, com o intuito de tornar o processo de elaboração da medida inteiramente transparente para outros pesquisadores (Epstein \& King, 2013). Dessa forma, percebe-se que uma medida confiável é elemento crucial para possibilitar a replicabilidade

9 "Se algum de nós pisasse na mesma balança de banheiro uma centena de vezes consecutivas, e se a balança estivesse funcionando de maneira confiável, ela nos daria o mesmo peso nas cem vezes consecutivas - mesmo se tal peso não fosse preciso" (Epstein \& King, 2013, p. 105). 
da pesquisa empírica, por isso, deve ser objeto de análise cuidadosa, tanto nos casos de análises quantitativas quanto nos casos de análise qualitativa da realidade (Epstein \& King, 2013).

Além disso, no que se refere à segunda dimensão, segundo Epstein e King (2013), "validade é a extensão à qual uma medida confiável reflete o conceito fundamental sendo medido" (p. 111). ${ }^{10}$ Em outras palavras, enquanto a confiabilidade está relacionada com a inalterabilidade, a validade está relacionada diretamente com a precisão das medidas realizadas. Assim, certificar-se de que uma medida é confiável nada diz sobre o quanto ela é válida.

Dessa forma, percebe-se que verificar a validade de uma medida é mais difícil do que verificar sua confiabilidade. Mesmo que nenhum procedimento de medida possa fornecer uma verdade exata da realidade, uma avaliação criteriosa da validade de uma medida pode ser fundamental para uma pesquisa empírica (Epstein \& King, 2013). Afinal, tendo em vista que todas as conclusões sobre o mundo são incertas, reconhece-se que até mesmo os conceitos de "certo" e "errado" são categorias probabilísticas (Epstein \& King, 2013).

Para Epstein e King (2013), a definição da validade de uma medida depende de três critérios: validade visual, imparcialidade e eficiência. Segundo os autores, nenhum desses critérios é sempre necessário e, ainda que estejam juntos, podem não ser suficientes (Epstein \& King, 2013). Não obstante, estes critérios se constituem como ferramentas úteis à tarefa de analisar se uma medida é mais ou menos válida (Epstein \& King, 2013).

Em primeiro lugar, para uma medida ser visualmente válida, ela deve ser consistente com evidência prévia - quantitativa, qualitativa e, até mesmo, impressionis-

10 "Mais cedo observamos que uma balança de banheiro era confiável se alguém subisse nela cem vezes consecutivas e obtivesse o mesmo valor. Isto, no entanto, não necessariamente quer dizer que a balança é válida. Se o peso real de alguém for 150 e a balança, mesmo uma centena de vezes consecutivas, relatar 125 , não daríamos valor à balança. É tal preocupação com a precisão que a validade implica” (...) “Uma balança que é não só confiável como também válida mostra um peso de 150 cem vezes consecutivas; uma balança que mostra 125 cem vezes consecutivas é confiável, mas não é válida" (Epstein \& King, 2013, p. 111). ta informal (Epstein \& King, 2013). A validade visual, contudo, não deve ser encarada como um julgamento casual de plausibilidade; ao invés disso, esse critério demanda uma comparação cuidadosa da nova medida com as evidências anteriores (Epstein \& King, 2013).

Em segundo lugar, além de serem visualmente válidas, segundo Epstein e King (2013), os procedimentos de medida devem ser aproximadamente imparciais. Isso ocorre quando eles produzem medidas que, em média, estão corretas após repetidas aplicações. Isso significa que, ao se aplicar o mesmo procedimento de medida a um grande número de temas, algumas vezes a medida será grande demais e, por outras, pequena demais, mas, em média, ela produzirá a resposta certa (Epstein \& King, 2013). ${ }^{11}$

Em terceiro lugar, diretamente relacionado à imparcialidade, o critério da eficiência é utilizado para se escolher entre várias medidas imparciais, dando preferência àquela com a menor variação (Epstein \& King, 2013). ${ }^{12}$ A eficiência, portanto, indica o grau de confiabilidade de medidas imparciais (Epstein \& King, 2013).

\subsection{Inovação Farmacêutica}

Para que se torne possível a total compreensão do desenvolvimento deste estudo, é necessário, também, o esclarecimento do conceito de inovação farmacêutica utilizado. Dessa forma, poder-se-á replicar o caminho investigativo realizado e, além disso, contestar os resultados encontrados neste trabalho de maneira mais precisa (Epstein \& King, 2013).

Não obstante a indústria farmacêutica comumente utilize o termo inovador para designar apenas uma

\footnotetext{
11 "Suponha que nós perguntamos a uma centena de pessoas para subirem em uma balança de banheiro. Nossa balança seria imparcial se cada pessoa que foi medida fosse mostrada como sendo um pouco mais pesada ou um pouco mais leve, mas os erros nos pesos mostrados fossem mínimos. Um exemplo de procedimento tendencioso seria pedir aos sujeitos que dissessem o seu próprio peso. Muito provavelmente, alguns dariam respostas precisas, ou respostas que fossem satisfatoriamente certas; outros reagiriam à situação social e subestimariam seus pesos. Já que às subestimadas não seriam canceladas por um conjunto similar de sobreestimadas, o resultado seria uma medida enviesada" (Epstein \& King, 2013, p. 117). 12 "Por exemplo, se tivéssemos acesso a duas balanças de banheiro, cada uma das quais imparcial, mas uma produzisse erros menores em uma medida, escolheríamos esta última" (Epstein \& King, 2013, p. 120).
} 
nova entidade química, segundo o conceito defendido por Angell (2007), um medicamento (invenção), para ser considerado inovador, deve ser avaliado em duas dimensões: a) em relação à novidade do composto em si (tipo químico) e b) em relação à probabilidade de que o medicamento apresente algum benefício superior aos medicamentos disponíveis no mercado. Em outras palavras, para ser considerado inovador, um medicamento deve ser uma nova entidade química e, além disso, possuir probabilidade de representar um aperfeiçoamento significativo, em comparação com os produtos já comercializados, no que se refere ao tratamento, ao diagnóstico ou à prevenção de uma doença (droga para inspeção prioritária) (Angell, 2007).

A definição de inovação farmacêutica defendida por Angell (2007), dentre as categorias elaboradas por Kesselheim, Wang e Avorn (2013), insere-se entre as definições que levam em consideração a avaliação do valor terapêutico dos novos medicamentos, como condição prévia de serem classificados como inovadores. Isso significa dizer que, ao assumir essa posição, a definição de Angell (2007) se diferencia daquelas que consideram a inovação farmacêutica a partir da contagem de novas drogas aprovadas, dos resultados econômicos obtidos ou do número de patentes concedidas.

Após um estudo sistemático sobre esses diferentes tipos de definição de inovação farmacêutica, KesseIheim, Wang e Avorn (2013) concluem que as definições que levam em consideração o valor terapêutico dos medicamentos são as mais adequadas para se avaliar a efetividade dos investimentos no desenvolvimento de novas drogas. Segundo os autores, tendo em vista a complexidade que envolve os resultados da inovação no setor farmacêutico, não seria adequado fazer uso de definições simplistas para compreender esse fenômeno, como, por exemplo, por meio da mera contagem de novas drogas aprovadas por agências reguladoras (Kesselheim, Wang \& Avorn, 2013).

De acordo com Kesselheim, Wang e Avorn (2013), nesse sentido, a utilização de concepções reducionistas de inovação farmacêutica pode causar sérios danos à elaboração de políticas públicas na área da saúde. Como exemplo, os autores relatam a experiência estadunidense com a elaboração do US Prescription Drug User Fee Act, de 1992, que, com o intuito de incentivar a inovação farmacêutica, independentemente de seu respectivo valor terapêutico, estabeleceu medidas voltadas à aceleração da aprovação de novas drogas. Todavia, como resultado dessas medidas, observou-se que uma parcela substancial dos novos medicamentos aprovados não só oferecia um avanço marginal em relação ao estado da arte farmacêutico, mas também apresentavam sérios problemas de segurança (Kesselheim, Wang \& Avorn, 2013).

Dessa forma, considerando a complexa realidade da inovação farmacêutica e seus importantes reflexos socioeconômicos no contexto nacional, no âmbito da presente investigação, optou-se pela utilização de uma definição mais completa de inovação farmacêutica (Angell, 2007), que leva em consideração o valor terapêutico dos novos medicamentos aprovados, com o intuito de avaliar, empiricamente, se o número de pedidos de patentes depositados pode se configurar como medida confiável e válida do desempenho inovador do setor farmacêutico no Brasil.

Ao se adotar esse pressuposto teórico para a delimitação do conceito de inovação no setor farmacêutico, poder-se-ia argumentar que, por demandar uma análise qualitativa, tendo em conta a exigibilidade de se avaliar o ganho terapêutico, seria inadequado atrelar a análise quantitativa a esse conceito qualitativo. Todavia, o objetivo dessa pesquisa consiste em testar a confiabilidade e a validade de se adotarem os depósitos de pedidos de patente como necessariamente um avanço na pesquisa e no desenvolvimento de processos e produtos.

\subsection{Doença de Chagas}

Essa investigação faz parte de um diagnóstico empírico mais amplo sobre os efeitos do sistema jurídico de patentes e o caso das doenças negligenciadas, no qual serão estudadas, detalhadamente, a realidade de todas essas enfermidades - definidas de acordo com a lista presente na resolução $n^{\circ} 80 / 2013$, do INPI (Resolução $n^{\circ} 80$ do Instituto Nacional da Propriedade Industrial de 19 de março de 2013). Com o intuito de evitar qualquer viés na realização desse diagnóstico, decidiu-se também que as doenças seriam analisa- 
das de acordo com a ordem disposta na própria resolução (Resolução $n^{\circ} 80$ do Instituto Nacional da Propriedade Industrial de 19 de março de 2013). Assim, a execução do referido diagnóstico obedeceria a uma lógica objetiva e não a algum tipo de preferência dos pesquisadores; reduzindo-se, ao máximo, o nível de interferência humana nas escolhas realizadas (Epstein \& King, 2013). Portanto, a doença de Chagas, por ser a primeira dessa lista (Resolução $n^{\circ} 80$ do Instituto Nacional da Propriedade Industrial de 19 de março de 2013), foi escolhida como o objeto inicial deste diagnóstico e, consequentemente, como objeto da presente investigação.

Poder-se-ia objetar, ademais, que as inferências realizadas neste trabalho não poderiam se basear apenas na análise dos pedidos de patente relacionados à doença de Chagas, uma vez que não se poderia generalizar a experiência observada na realidade de uma única enfermidade à realidade de todas as doenças negligenciadas e, menos ainda, à inovação farmacêutica na realidade brasileira, de modo geral. Contudo, entende-se que, ao se demonstrar, fundamentadamente, em uma única ocasião, a invalidade de um procedimento de medida, estar-se-á demonstrando sua invalidade de modo geral.

Segundo Epstein e King (2013), o ato de medida consiste em "comparar um objeto de estudo (por exemplo, um evento, sujeito ou processo do mundo real) com algum standard (padrão), como aqueles existentes para quantidades, capacidades ou categorias" (pag. 101). Assim sendo, a partir do argumento utilizado pelos próprios autores, de nada serviria uma balança de banheiro que apenas medisse alguns casos e não funcionasse em outros (Epstein \& King, 2013). Isso estaria, portanto, completamente avesso à própria essência de um procedimento de medida.

Por outro lado, no caso de os resultados encontrados não corroborarem a hipótese inicial deste trabalho e o número de pedidos de patente depositados puder ser considerado uma medida válida de inovação farmacêutica no caso da doença de Chagas, reconhece-se a impossibilidade de se inferir, categoricamente, a validade desse procedimento de medida de um modo geral. Afinal, o fato de esta medida se consti- tuir validamente, no caso da doença de Chagas, não implica sua validade em todos os outros casos. Nessa situação, especificamente, seria necessária uma pesquisa mais abrangente e com um maior número de enfermidades envolvidas.

\subsection{Coleta de Dados}

Com o objetivo de realizar um levantamento do número de depósitos de pedidos de patente, inicialmente, acessou-se a página oficial do INPI (http:// www.inpi.gov.br/) na intenet, clicando-se, sucessivamente, nos respectivos links do lado esquerdo da tela: "Serviços", "Patentes" e, por fim, "Busca". Na página seguinte, o usuário pode optar por realizar o login ou pesquisar anonimamente. Ambas as opções redirecionam para a página "Consulta à Base de Dados do INPI", que é a base de dados utilizada neste trabalho (INPI, 2017).

Posteriormente, com o intuito de permitir o alcance do maior número de depósitos possível, selecionou-se a alternativa "Patente", digitou-se a palavra-chave "Chagas", no espaço em branco ao lado, e escolheu-se a opção "resumo". A partir desse procedimento, encontraram-se 65 depósitos e, após a coleta e a leitura integral dos resumos dos pedidos de patente, foram retirados do corpo de análise aqueles que não tinham relação com o objeto de estudo desta investigação, selecionando-se somente aqueles que estavam associados diretamente à doença de Chagas.

Dessa maneira, os seguintes depósitos foram descartados: "BR 112012028451 7", composição tópica iodopovidona, uma vez que neste depósito o termo "Chagas" foi utilizado apenas como sinônimo de ferimento; "BR 102014028172 0", kit e método para imunodiagnóstico das leishmanioses e uso de uma proteína de leishmania e de um peptídeo derivado, e “BR 102014004107 9", método e kit para diagnóstico das leishmanioses utilizando peptídeos sintéticos, pois, em ambos os casos, a doença de chagas foi utilizada apenas como exemplo de ausência de ação cruzada com agentes etiológicos de outras doenças; "PI 9703992-6", equipamento pedagógico para ensino de parasitologia, visto que este depósito tem como objetivo auxiliar professores no ensino de algumas doenças, sendo, apenas, um material de apoio di- 
dático; “PI 9803083-3 A2”, guilhotina para corte de tubérculos, porque no depósito em questão o termo chagas refere-se tão somente a um determinado tipo de corte realizado no caule de uma árvore.

A fim de controlar a eventual duplicidade de entradas de um mesmo pedido, de apresentar os dados a serem analisados de maneira mais adequada e de possibilitar a replicabilidade da pesquisa (Epstein \& King, 2013), após a coleta de todos os pedidos relativos à doença de Chagas, foi estabelecida uma tabela unificada, na qual os pedidos de patente estão identificados por seus respectivos números e encontram-se organizados em ordem cronológica decrescente, de acordo com as datas em que foram depositados no INPI. Além disso, nessa tabela, pode-se verificar a data da publicação dos pedidos, seus respectivos status e a finalidade da invenção a que dizem respeito.

Também, para que fosse possível uma compreensão mais ampla e geral das informações recolhidas, produziu-se um gráfico de colunas, em que o eixo horizontal e o eixo vertical representam o status dos depósitos no momento da coleta e o número de depósitos encontrados em cada categoria, respectivamente. É importante evidenciar que a escolha do gráfico de colunas se deu pela sua finalidade de comparar itens, fato relevante para a construção das implicações desenvolvidas ao longo do trabalho.

\subsection{Limitações}

É importante ressaltar que a atualização da base de dados do INPI (2017) pode apresentar certa morosidade, sobretudo, no que se refere aos depósitos de origem internacional. Com base nas normas dispostas na resolução N $77 / 2013$ do INPI (Resolução $n^{\circ} 77$ do Instituto Nacional da Propriedade Industrial de 18 de março de 2013), percebe-se que os depósitos de pedido de patente somente serão anexados à base de dados oficial após a apresentação de petição de requerimento, referente à entrada na fase nacional do pedido internacional depositado através do Tratado de Cooperação de Patentes (Tratado de Cooperação em Matéria de Patentes, de 9 de junho de 1970).

Dessa maneira, é possível que se encontre, nessa base de dados, utilizando-se os mesmos procedimentos metodológicos, depósitos que não foram apresenta- dos aqui. No momento da coleta, pois, tais pedidos poderiam ainda não ter sido somados à base, ainda que já tivessem sido depositados internacionalmente.

\section{Depósitos de pedidos de patente relacionados à doença de chagas}

Com a intenção de possibilitar a replicabilidade da pesquisa realizada (Epstein \& King, 2013), na Tabela 1, pode-se verificar detalhadamente a totalidade de pedidos de patente depositados no INPI relacionados à doença de Chagas. Os pedidos de patente estão identificados por seus respectivos números e encontram-se organizados em ordem cronológica decrescente, de acordo com as datas em que foram depositados no INPI. Além disso, na tabela, também é possível verificar o status do pedido de patente (arquivado, concedido, deferido, desistido, em andamento, extinto ou indeferido) e a finalidade da invenção a ser patenteada (prevenção, diagnóstico ou tratamento).

De acordo com a tabela, percebe-se que, em toda a história de atuação do INPI, foram depositados 60 pedidos de patente relacionados à doença de Chagas tendo o primeiro deles ocorrido em 1991 e o último, em $2015^{13}$. Ademais, em uma análise mais detida dos dados coletados, chama a atenção o fato de pedidos de patente depositados há bastante tempo ainda estarem em andamento, como é o caso, por exemplo, dos pedidos “PP 1101127-0" e "PI 0201237-5”, que foram depositados, aproximadamente, há 20 e 15 anos. ${ }^{14}$

13 Comparativamente, é importante ressaltar o estudo realizado por Silva e Luz Júnior (2016), no qual foram encontrados apenas 43 depósitos de pedidos de patente relacionados à doença de Chagas, ao longo da história de atuação do INPI. Essa distinção se deu (i) em função da diferença das palavras-chave pesquisadas e (ii) em função do lapso temporal utilizado para a coleta de dados. Enquanto, neste artigo, buscou-se apenas pelo termo "Chagas" no resumo, para encontrar o maior número de depósitos possível, Silva e Luz Júnior (2016) utilizaram os termos "doença and chagas" e "Trypanosoma cruzi and diagnosis". Além disso, enquanto, neste artigo, a coleta de dados foi feita em 07 de julho de 2017, a coleta de Silva e Luz Júnior (2016) foi feita em 10 de junho de 2015 - aproximadamente, 2 anos e 1 mês de diferença. 14 De acordo com o estudo realizado por Jannuzzi e Vasconcellos (2013), acerca da concessão de patentes de medicamentos, verificou-se que o tempo médio para a concessão de uma patente farmacêutica é 11,5 anos no Brasil. Segundo os autores, a demora de mais de 10 anos na concessão de patentes de medicamentos tem levado a dilatação do prazo de vigência das mesmas, com implicações negativas diretas para a entrada de novos medicamentos genéricos no mercado brasileiro (Jannuzzi; Vasconcellos, 2013). 
Tabela 1. Depósitos de pedidos de patente publicados pelo INPI relacionados à doença de Chagas

\begin{tabular}{|c|c|c|c|c|c|}
\hline & Número do pedido & Data do depósito & Data da publicação & Status do pedido & Finalidade da Invenção \\
\hline 1 & $\begin{array}{l}\text { BR } 102015028438 \\
1\end{array}$ & $06 / 11 / 2015$ & 09/05/2017 & Em andamento & Tratamento \\
\hline 2 & $\begin{array}{l}\text { BR } 102014003374 \\
2\end{array}$ & $13 / 02 / 2014$ & $20 / 09 / 2016$ & Em andamento & Tratamento \\
\hline 3 & $\begin{array}{l}\text { BR } 102013031927 \\
9\end{array}$ & $12 / 12 / 2013$ & 06/10/2015 & Em andamento & Tratamento \\
\hline 4 & $\begin{array}{l}\text { BR } 102013028096 \\
8\end{array}$ & $31 / 10 / 2013$ & 08/09/2015 & Em andamento & Tratamento \\
\hline 5 & $\begin{array}{l}\text { BR } 102013026558 \\
6\end{array}$ & $15 / 10 / 2013$ & $28 / 07 / 2015$ & Em andamento & Prevenção \\
\hline 6 & $\begin{array}{l}\text { BR } 102013017357 \\
6\end{array}$ & 05/07/2013 & $31 / 05 / 2016$ & Em andamento & Diagnóstico \\
\hline 7 & $\begin{array}{l}\text { BR } 102013017358 \\
4\end{array}$ & 05/07/2013 & $20 / 09 / 2016$ & Em andamento & Diagnóstico \\
\hline 8 & $\begin{array}{l}\text { BR } 102013002127 \\
0\end{array}$ & $22 / 01 / 2013$ & $29 / 10 / 2014$ & Arquivado & Tratamento \\
\hline 9 & $\begin{array}{l}\text { BR } 102012027997 \\
5\end{array}$ & $31 / 10 / 2012$ & $21 / 10 / 2014$ & Em andamento & Diagnóstico \\
\hline 10 & $\begin{array}{l}\text { BR } 112014009846 \\
8\end{array}$ & $24 / 10 / 2012$ & $18 / 04 / 2017$ & Em andamento & Prevenção \\
\hline 11 & $\begin{array}{l}\text { BR } 102012019095 \\
8\end{array}$ & $31 / 07 / 2012$ & $13 / 01 / 2015$ & Arquivado & Tratamento \\
\hline 12 & $\begin{array}{l}\text { BR } 102012019428 \\
7\end{array}$ & $27 / 06 / 2012$ & $28 / 04 / 2015$ & Em andamento & Tratamento \\
\hline 13 & $\begin{array}{l}\text { BR } 102012018214 \\
9\end{array}$ & $21 / 06 / 2012$ & $19 / 08 / 2014$ & Arquivado & Prevenção \\
\hline 14 & $\begin{array}{l}\text { BR } 102012010303 \\
6\end{array}$ & $02 / 05 / 2012$ & 07/04/2015 & Em andamento & Prevenção \\
\hline 15 & $\begin{array}{l}\text { BR } 102012001876 \\
4\end{array}$ & $27 / 01 / 2012$ & $12 / 11 / 2013$ & Em andamento & Prevenção \\
\hline 16 & PI 1104815-8 & $31 / 10 / 2011$ & $12 / 01 / 2016$ & Em andamento & Diagnóstico \\
\hline 17 & PI 1106302-5 & $24 / 10 / 2011$ & $22 / 12 / 2015$ & Arquivado & Tratamento \\
\hline 18 & PI 1103777-6 & $19 / 08 / 2011$ & $30 / 07 / 2013$ & Em andamento & Tratamento \\
\hline 19 & PI 1102567-0 & 06/05/2011 & $30 / 07 / 2013$ & Em andamento & Tratamento \\
\hline 20 & PI 0904827-8 & $06 / 11 / 2009$ & $12 / 07 / 2011$ & Em andamento & $\begin{array}{l}\text { Diagnóstico e Preven- } \\
\text { ção }\end{array}$ \\
\hline 21 & PI 0903451-0 & $27 / 08 / 2009$ & $10 / 05 / 2011$ & Em andamento & Tratamento \\
\hline
\end{tabular}




\begin{tabular}{|c|c|c|c|c|c|}
\hline 22 & PI 0805492-4 & $02 / 12 / 2008$ & $24 / 08 / 2010$ & Em andamento & Tratamento \\
\hline 23 & PI 0805753-2 & $31 / 10 / 2008$ & $17 / 04 / 2012$ & Em andamento & Prevenção \\
\hline 24 & PI 0801906-1 & $15 / 01 / 2008$ & $01 / 02 / 2011$ & Em andamento & Prevenção \\
\hline 25 & PI 0806285-4 & $15 / 01 / 2008$ & $11 / 01 / 2011$ & Em andamento & Prevenção \\
\hline 26 & PI 0714722-8 & $13 / 07 / 2007$ & $26 / 03 / 2013$ & Arquivado & Tratamento \\
\hline 27 & PI 0700836-8 & $20 / 03 / 2007$ & $04 / 11 / 2008$ & Em andamento & $\begin{array}{l}\text { Tratamento e preven- } \\
\text { ção }\end{array}$ \\
\hline 28 & PI 0709560-0 & $14 / 03 / 2007$ & $19 / 07 / 2011$ & Em andamento & $\begin{array}{l}\text { Prevenção e Trata- } \\
\text { mento }\end{array}$ \\
\hline 29 & PI 0700314-5 & $16 / 01 / 2007$ & $02 / 09 / 2008$ & Arquivado & Prevenção \\
\hline 30 & PI 0603871-9 & $24 / 08 / 2006$ & $15 / 04 / 2008$ & Em andamento & Tratamento \\
\hline 31 & PI 0603872-7 & $24 / 08 / 2006$ & $15 / 04 / 2008$ & Em andamento & $\begin{array}{l}\text { Diagnóstico e Trata- } \\
\text { mento }\end{array}$ \\
\hline 32 & PI 0600759-7 & $10 / 03 / 2006$ & $19 / 12 / 2006$ & Concedido & Diagnóstico \\
\hline 33 & PI 0502172-3 & $13 / 06 / 2005$ & $30 / 01 / 2007$ & Em andamento & Tratamento \\
\hline 34 & PI 0417231-0 & $06 / 12 / 2004$ & $17 / 04 / 2007$ & Arquivado & Diagnóstico \\
\hline 35 & PI 0401107-4 & $13 / 04 / 2004$ & $22 / 11 / 2005$ & Em andamento & $\begin{array}{l}\text { Tratamento e Preven- } \\
\text { ção }\end{array}$ \\
\hline 36 & PI 0310123-1 & $04 / 12 / 2003$ & $25 / 10 / 2005$ & Em andamento & Diagnóstico \\
\hline 37 & PI 0305933-2 & $02 / 12 / 2003$ & 06/09/2005 & Concedido & Prevenção \\
\hline 38 & PI 0315200-6 & $10 / 10 / 2003$ & $16 / 08 / 2005$ & Arquivado & Diagnóstico \\
\hline 39 & PI 0211026-1 & $21 / 06 / 2002$ & $10 / 08 / 2004$ & Arquivado & Tratamento \\
\hline 40 & PI 0202214-1 & 05/06/2002 & $24 / 09 / 2002$ & Em andamento & $\begin{array}{l}\text { Diagnóstico e Preven- } \\
\text { ção }\end{array}$ \\
\hline 41 & PI 0201237-5 & $25 / 03 / 2002$ & $02 / 12 / 2003$ & Em andamento & Tratamento \\
\hline 42 & PI 0206501-0 & $17 / 01 / 2002$ & $13 / 01 / 2004$ & Deferido & Tratamento \\
\hline 43 & PI 0000886-9 & $13 / 01 / 2000$ & $11 / 09 / 2001$ & Arquivado & Diagnóstico \\
\hline 44 & PI 9903472-7 & $13 / 08 / 1999$ & $24 / 04 / 2001$ & Indeferido & Tratamento \\
\hline
\end{tabular}




\begin{tabular}{|c|c|c|c|c|c|}
\hline 45 & PI 9903250-3 & 30/07/1999 & 09/05/2000 & Arquivado & Diagnóstico \\
\hline 46 & PI 9813637-2 & 04/12/1998 & $04 / 12 / 2001$ & Arquivado & Prevenção \\
\hline 47 & PI 9805072-9 & $27 / 10 / 1998$ & $06 / 06 / 2000$ & Arquivado & Tratamento \\
\hline 48 & PI 9803243-7 & $27 / 08 / 1998$ & $21 / 03 / 2000$ & Indeferido & Tratamento \\
\hline 49 & PP $1101127-0$ & $14 / 05 / 1997$ & $10 / 11 / 1998$ & Em andamento & $\begin{array}{l}\text { Diagnóstico e preven- } \\
\text { ção }\end{array}$ \\
\hline 50 & PP $1100552-1$ & $13 / 05 / 1997$ & - & - & - \\
\hline 51 & PP $1100553-0$ & $13 / 05 / 1997$ & - & - & - \\
\hline 52 & PI 9700266-6 & $18 / 02 / 1997$ & 03/11/1998 & Indeferido & Diagnóstico \\
\hline 53 & PI 9611455-0 & $14 / 11 / 1996$ & $02 / 01 / 2001$ & Extinto & $\begin{array}{l}\text { Diagnóstico e preven- } \\
\text { ção }\end{array}$ \\
\hline 54 & PI 9603267-7 & 02/08/1996 & 28/04/1998 & Arquivado & Diagnóstico \\
\hline 55 & PI 9602428-3 & 17/05/1996 & 06/10/1998 & Arquivado & Prevenção \\
\hline 56 & PI 9503451-0 & 26/07/1995 & $30 / 09 / 1997$ & Arquivado & Diagnóstico \\
\hline 57 & PI 9507182-2 & 20/03/1995 & $14 / 10 / 1997$ & Extinto & Diagnóstico \\
\hline 58 & PI 9400950-3 & 07/04/1994 & 07/11/1995 & Extinto & Diagnóstico \\
\hline 59 & PI 9305995-7 & $11 / 02 / 1993$ & $11 / 03 / 1997$ & Desistido & $\begin{array}{l}\text { Diagnóstico e preven- } \\
\text { ção }\end{array}$ \\
\hline 60 & PI 9104287-9 & 03/10/1991 & 20/04/1993 & Extinto & Diagnóstico \\
\hline
\end{tabular}

Fonte: Dados coletados pelos autores na base de dados oficial do INPI, 2017

* A última atualização dos dados coletados ocorreu em 07 de julho de 2017.

Notas:

${ }^{1}$ Não foi possível acessar o conteúdo do pedido "PP 1100552-1" - Antígenos recombinantes de Trypanosoma cruzi. Ao tentar abrir as informações deste depósito na base de dados do INPI (2017), a página seguinte se encontrava parcialmente em branco seguida de uma mensagem de erro, em função de problemas no sítio do INPI (http://www.inpi.gov.br/), impedindo o acesso aos dados.

${ }^{2}$ Não foi possível acessar o conteúdo do pedido "PP 1100553-0" Kit para o diagnóstico imunológico da doença de Chagas usando antígenos recombinantes conjugados a uma atividade enzimática" pela mesma razão citada acima. 
Ainda no que se refere à análise dos dados trazidos pela tabela, é possível perceber uma variação considerável do status em que se encontram os pedidos de patente. Por isso, com o intuito de facilitar a visualização dos dados relativos a essa informação, elaborou-se o gráfico, a seguir.

\section{Gráfico 1. Status dos pedidos de patente}

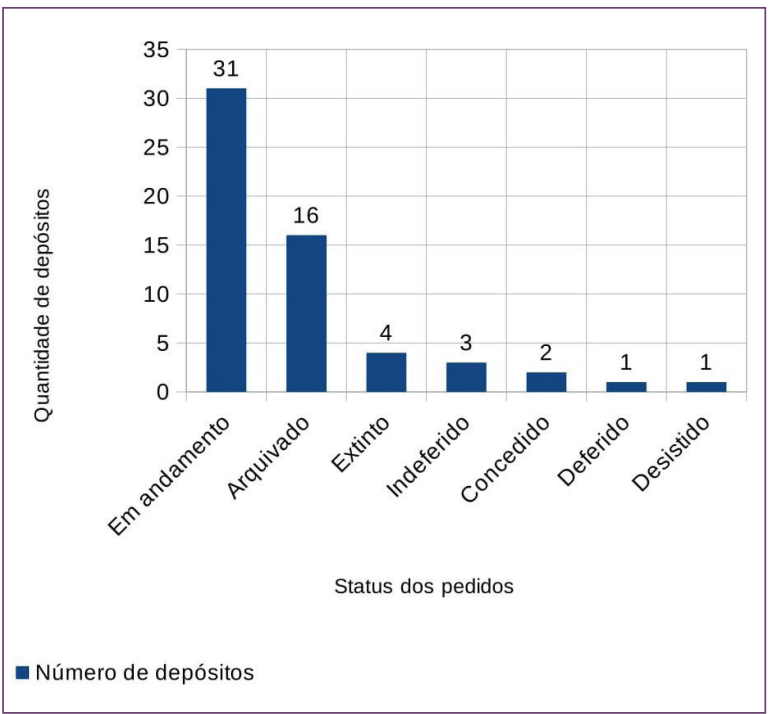

Nesse contexto, para que seja possível uma compreensão mais detalhada dos dados coletados, o gráfico acima apresenta, especificamente, os pedidos de patentes organizados pelo status em que se encontravam na época da coleta - excluindo-se os pedidos "PP 1100552-1" e "PP 1100553-0", que não puderam ter seus conteúdos acessados (ver tabela). Assim, a partir dos dados expostos acima, dos 58 pedidos analisados, observa-se que a expressiva maioria (31) ainda se encontra em andamento ${ }^{15} ; 16$ se encontram arquivados ${ }^{16}$; 4 se encontram extintos ${ }^{17} ; 3$ se encon-

15 Os pedidos em andamento são aqueles cujo processo está na fase de publicação, na fase de análise da documentação ou na fase da análise de anuência prévia. De modo geral, são aqueles que ainda estão tramitando, porém não tiveram o julgamento final dos requisitos necessários para a obtenção da exclusividade (Lei n 9279, de 14 de maio, 1996).

160 arquivamento de pedidos ocorrerá se o depositante não requerer o exame do pedido de patente, dentro do prazo legal, ou não responder alguma exigência realizada pelo INPI, de acordo com os artigos 33, 34 e 36 da LPI (Lei n 9279, de 14 de maio, 1996). 17 A extinção pode se dar pela expiração do prazo de vigência da patente, pela renúncia de seu titular, pela caducidade, pela falta de pagamento da retribuição anual, nos prazos previstos no $\S 2^{\circ}$ do art. 84 e no art. 87 da LPI. Também pode ocorrer a extinção pela tram indeferidos ${ }^{18} ; 2$ se encontram concedidos $^{19} ; 1$ se encontra deferido ${ }^{20} ; 1$ se encontra desistido. ${ }^{21}$

Em um primeiro momento, a partir dos dados analisados no gráfico, é possível observar, ainda que preliminarmente, um indicativo de ineficiência da atual conformação do sistema de patentes, especificamente, no que diz respeito à organização administrativa dos seus trâmites internos. O baixo número de pedidos de patente concedidos (2), deferidos (1) e indeferidos (3) pelo INPI e o alto número de pedidos em andamento - podendo atingir até 20 anos de tramitação (ver tabela) -, evidenciam, com efeito, uma desproporção existente entre o tempo despendido e os resultados da operacionalização cotidiana do sistema.

Como mencionado acima, trata-se de uma apreciação preliminar sobre o tema, uma vez que este não está diretamente relacionado ao escopo principal desta investigação. É possível afirmar apenas que esses dados, em linha de princípio, compõem um quadro indicativo de ineficiência do sistema patentário - que pode gerar efeitos negativos na pesquisa, no desenvolvimento e na distribuição de novos medicamentos para a população (Jannuzzi \& Vasconcellos, 2013, 2017) -, sendo necessária, posteriormente, uma análise específica, qualitativa e mais detalhada dos dados coletados.

Para a finalidade principal deste trabalho, contudo, é relevante destacar, primordialmente, o baixíssimo

ausência de procurador devidamente qualificado e domiciliado no país, com poderes para representação administrativa e judicial, inclusive para receber citações, no caso de pessoa domiciliada no exterior (Lei n 9279, de 14 de maio, 1996).

180 indeferimento ocorre quando o depositante não se manifesta sobre o parecer desfavorável, não cumpre, de modo satisfatório, às exigências emitidas, se as razões que fundamentam suas manifestações forem consideradas como improcedentes ou, ainda, se as emendas apresentadas junto com a manifestação forem consideradas insuficientes para obter o privilégio pretendido (Lei $n^{\circ}$ 9279, de 14 de maio, 1996).

19 Os pedidos concedidos são aqueles que foram deferidos e que o pagamento da retribuição para expedição da carta-patente foi realizado (Lei n 9279, de 14 de maio, 1996).

20 É a invenção que, além de não possuir qualquer proibição legal, após a realização do exame, está completa, atende às formalidades estabelecidas pelo INPI e contempla os requisitos de patenteabilidade (Lei $n^{\circ}$ 9279, de 14 de maio, 1996).

21 A desistência ocorre quando o depositante pede o encerramento do processo em andamento (Lei n 9279, de 14 de maio, 1996). 
número de pedidos de patente concedidos (2) e deferidos (1), em relação aos outros pedidos depositados (58). Isso significa dizer que, até a data de coleta dos dados dessa pesquisa, de todos os pedidos de patente relacionados à doença de Chagas (58), em mais de 24 anos, apenas 2 foram concedidos e 1 deferido pelo INPI - tendo preenchido, portanto, todos os requisitos de patenteabilidade. ${ }^{22}$

De acordo com o artigo $8^{\circ}$, da Lei de Propriedade Industrial (LPI) (Lei $n^{\circ}$ 9279, de 14 de maio, 1996), uma invenção, para que seja patenteável, deve atender aos requisitos da novidade ${ }^{23}$, da atividade inventiva ${ }^{24}$ e da aplicação industrial ${ }^{25}$. Isso significa dizer que, no contexto apresentado, apenas $3(5,17 \%)$ pedidos cumpriram, necessariamente, os requisitos legais supramencionados. Os outros 55 pedidos, que se encontram em andamento, arquivados, extintos, indeferidos ou desistidos, não podem ser considerados, necessariamente, invenções que detenham novidade, atividade inventiva e aplicação industrial.

\section{Os depósitos de pedidos de patente e a medida da inovação farmacêutica}

Considerando os objetivos iniciais propostos pela presente investigação, a partir dos dados expostos na seção anterior (tabela e gráfico), faz-se necessário verificar, especificamente, se o número de depósitos

22 É necessário destacar, todavia, a necessidade de se fazer um estudo qualitativo mais aprofundado para se averiguar as razões que fundamentam a extinção dos 4 pedidos de patente extintos, pois, no caso de a extinção ter sido motivada pela expiração do prazo de vigência de uma patente concedida, seria correto incluí-los no grupo de patentes que, ao longo da história do INPI, preencheram os requisitos de patenteabilidade. Apesar de não ser a única possibilidade de extinção de um pedido de patente, essa deve ser uma questão analisada em uma investigação qualitativa posterior, para que se possa alcançar uma maior precisão dos resultados. Por enquanto, essa questão será admitida, para os fins da presente investigação, como uma limitação do processo quantitativo de pesquisa escolhido. 23 Segundo o artigo 11, da LPI, uma invenção é considerada nova quando não está compreendida no estado da técnica (Lei ${ }^{\circ}$ 9279, de 14 de maio, 1996).

24 Segundo o artigo 13, da LPI, uma invenção "é dotada de atividade inventiva sempre que, para um técnico no assunto, não decorra de maneira evidente ou óbvia do estado da técnica" (Lei n 9279, de 14 de maio, 1996).

25 Segundo o artigo 15, da LPI, uma invenção é considerada suscetível de aplicação industrial quando possa ser utilizada ou produzida em qualquer tipo de indústria (Lei n 9279, de 14 de maio, 1996). de pedido de patente, relacionados à determinada doença, constitui-se como medida confiável e válida para se aferirem os níveis de inovação farmacêutica na realidade brasileira.

No que se refere à confiabilidade da medida analisada, tendo em vista o conceito elaborado por Epstein e King (2013), caso a coleta dos depósitos, em base oficial do INPI (2017), seja norteada por regras precisas, transparentes e replicáveis - de maneira que essa medida possa produzir o mesmo resultado várias vezes, independentemente de quem a estiver realizando -, é possível afirmar que o número de pedidos de patente, relacionados à determinada doença, pode ser considerado uma medida confiável da inovação farmacêutica.

Em outras palavras, como se poderia esperar, apesar de não ser uma medida necessariamente confiável, a confiabilidade dependerá, em sua essência, da conduta do pesquisador. Para que a confiabilidade da medida não seja prejudicada, segundo Epstein e King (2013), é necessário que o julgamento e a interpretação humana sejam reduzidos ao máximo em sua elaboração. E, nos casos em que for imprescindível, regras precisas sobre os procedimentos adotados devem ser elaboradas, com a intenção de tornar o processo de elaboração da medida inteiramente transparente para outros pesquisadores (Epstein \& King, 2013).

No caso específico dos pedidos de patente, a existência de uma base de dados oficial, a do INPI (2017), é um importante elemento para se garantir a confiabilidade da medida. Entretanto, alguns cuidados devem ser especialmente tomados, como, por exemplo, a definição clara das palavras-chave utilizadas para a pesquisa, a devida justificativa de descarte dos depósitos excluídos da análise (por não estarem diretamente relacionados com o objeto do estudo), o registro criterioso dos problemas apresentados pela base de dados do INPI (2017) (especialmente, no que se refere à ocultação acidental de conteúdo dos depósitos) e a indicação precisa do lapso temporal escolhido para se realizar a coleta (com o intuito de se evitarem as variações naturais de uma base de dados, com o advento de novos depósitos ou com o andamento processual dos depósitos já existentes). 
Apesar de o número de depósitos de pedido de patente, relacionados à determinada doença, poder ser considerado uma medida confiável - caso sejam tomados alguns cuidados específicos -, isso não significa que será também uma medida válida para se aferir os níveis de inovação farmacêutica na realidade brasileira, tendo em vista os conceitos elaborados por Epstein e King (2013). A medida em estudo, para ser considerada válida, deve ser analisada sob a orientação de três critérios: validade visual, imparcialidade e eficiência (Epstein \& King, 2013).

Para se verificar, em primeiro lugar, a observância do critério da validade visual, é necessária uma análise cuidadosa de evidências anteriores, de modo que não seja apenas um julgamento superficial de plausibilidade (Epstein \& King, 2013). Neste contexto, percebe-se, preliminarmente, que há um contraste entre a quantidade de depósitos relacionados à doença de Chagas (60) e as possibilidades de prevenção, de diagnóstico e de tratamento dessa enfermidade disponíveis (MSF, 2013). Segundo os Médicos Sem Fronteiras (2013), por exemplo, atualmente, existem apenas dois medicamentos disponíveis para o tratamento da doença de Chagas. ${ }^{26}$ Assim sendo, pode-se dizer que, se a inovação farmacêutica fosse medida a partir do número de depósitos de pedidos de patente, seriam encontrados resultados muito superiores do que se pode observar na realidade. Em outras palavras, ainda que preliminarmente, esse tipo de medida contrastaria com os níveis de inovação farmacêutica apontados em evidências anteriores, não representando o atual contexto de escassez existente em torno desta doença (Cruz et al., 2016) (Feres \& Silva, 2017) (Martins-Melo et al, 2014) (Pedrique et al, 2013).

26 De acordo com os Médicos Sem Fronteiras (2013), no Brasil, existe apenas o medicamento Benzonidazol (BNZ), enquanto em outros lugares do mundo, pode-se encontrar também o Nifurtimox. Um estudo posterior, realizado por Cruz et al. (2016), todavia, aponta que o medicamento Nifurtimox teve seu uso iterrompido por apresentar efeitos secundários. Segundo os autores, ademais, mesmo o BNZ sendo o único medicamento utilizado para o tratamento de Chagas, ele ainda apresenta algumas restrições importantes, "como a baixa eficácia na fase crônica da doença, a alta taxa de abandono do tratamento devido aos efeitos colaterais causados pelo medicamento, a de apresentar longo período de tratamento (entre 30 a 60 dias) e a inexistência de formulação pediátrica" (Cruz et al., 2016, p. 3).
Entre as enfermidades consideradas negligenciadas, a doença de Chagas é a que mais mata no Brasil (DNDi, 2017). Além disso, por ser uma doença negligenciada e contar com o desinteresse do setor farmacêutico, evidências anteriores demonstram uma grave falha de pesquisa e desenvolvimento de produtos farmacêuticos para a doença de Chagas (Cruz et al., 2016) (Feres \& Silva, 2017) (Martins-Melo et al, 2014) (Pedrique et al, 2013). Se o número de pedidos fosse uma medida de inovação visualmente válida, não haveria, prima facie, uma contradição entre seus resultados e a escassez apresentada pela real situação da enfermidade em estudo. Deste modo, a partir do descompasso apresentado acima, é possível afirmar que o número de depósitos de pedidos de patente, relacionados à determinada doença, não se constitui como uma medida visualmente válida para se aferir os níveis de inovação farmacêutica na realidade brasileira.

Ainda na dimensão da validade, uma medida precisa ser avaliada em relação ao critério da imparcialidade (Epstein \& King, 2013), segundo o qual, ao se aplicar o mesmo procedimento de medida a um grande número de temas, em média, ela deverá produzir a resposta certa (Epstein \& King, 2013). Como o conceito de inovação farmacêutica adotado exige, em primeiro lugar, que um medicamento (invenção) deva ser uma nova entidade química (Angell, 2007), de acordo com os dados analisados, percebe-se que o número de pedidos de patente que representam, comprovadamente, uma invenção patenteável, ao longo de mais de duas décadas, é bastante reduzido (3). Significa dizer, portanto, que quase a totalidade dos depósitos (55) não pode ser considerada, a priori, indicador do primeiro requisito de inovação farmacêutica, uma vez que não cumpriram os requisitos mínimos de patenteabilidade e, por isso, não podem ser considerados novas entidades químicas.

Além disso, no caso estudado, os depósitos concedidos e deferidos, mesmo tendo preenchido os requisitos mínimos de patenteabilidade de acordo com o INPI, não representam, necessariamente, sequer um novo medicamento. Apesar de estarem diretamente relacionados à doença de Chagas, esses pedidos podem representar qualquer tipo de invenção relacionada à prevenção, ao diagnóstico ou ao tratamento da doença. É necessária, assim, uma análise qualitativa posterior para se verificar a natureza de cada invenção. 
Com efeito, em razão do baixo número de pedidos de patente concedidos e deferidos, bem como da variabilidade dos tipos de invenções possíveis, percebe-se que o número de pedidos de patentes depositados não representa, diretamente, o desenvolvimento de novas entidades químicas. E, por isso, não serve para aferir, de maneira segura, o primeiro requisito da inovação farmacêutica.

Em relação ao segundo requisito de inovação farmacêutica trazido por Angell (2007), o medicamento deve possuir probabilidade de representar um aperfeiçoamento significativo, em comparação com os produtos já comercializados, no que se refere à prevenção, ao diagnóstico ou ao tratamento de uma doença. Além de não representar novas entidades químicas e nem mesmo, necessariamente, medicamentos, o número de pedidos de patente depositados, por ser uma medida estritamente quantitativa, não é capaz de avaliar, qualitativamente, o valor terapêutico dos novos medicamentos e, tampouco, de compará-los com os medicamentos já existentes no mercado. Por isso, não serve também para aferir, de maneira segura, o segundo requisito da inovação farmacêutica.

Desse modo, como o número de depósitos de pedidos de patentes não se constituem como formas seguras para se aferir o primeiro e o segundo requisitos de uma inovação farmacêutica (Angell, 2007), é possível afirmar que essa medida, além de visualmente inválida, não se constitui como uma medida imparcial (Epstein \& King, 2013).

No que se refere ao critério da eficiência, último critério de validade, não há maiores considerações a serem feitas. Uma vez que o critério da eficiência é utilizado para se escolher entre várias medidas imparciais - dando preferência para aquela com a menor variação -, no caso estudado, como a medida não pôde ser considerada imparcial, não há que se falar em um juízo de eficiência entre medidas imparciais (Epstein \& King, 2013). Assim sendo, afirma-se que o número de depósitos de pedidos de patente não é, óbvia e consequentemente, uma medida eficiente para se aferirem os níveis de inovação farmacêutica na realidade brasileira.

Por fim, a partir dos dados conhecidos analisados aci- ma - o baixo número de pedidos de patente concedidas e deferidas -, dos conceitos de confiabilidade e de validade das medidas (Epstein \& King, 2013) e do conceito de inovação farmacêutica adotado (Angell, 2007), infere-se, em primeiro lugar, que o número de depósitos de pedidos de patente, relacionados à determinada doença, pode ser considerado uma medida confiável da inovação farmacêutica, caso a coleta dos depósitos, em base oficial do INPI (2017), seja norteada por regras precisas, transparentes e replicáveis. De maneira que essa medida possa produzir o mesmo resultado várias vezes, independentemente de quem a estiver realizando. Em segundo lugar, infere-se que, apesar de confiável, por não ser considerada uma medida visual, imparcial e eficientemente válida, o número de depósitos de pedidos de patente, relacionados à determinada doença, não pode ser considerado uma medida válida para se aferirem os níveis de inovação farmacêutica na realidade brasileira.

\section{Conclusão}

Diante da pluralidade de entendimentos sobre o meIhor procedimento de medida a ser utilizado para se diagnosticar o desempenho da inovação farmacêutica, o objetivo inicial da presente investigação era o de verificar, especificamente, se o número de depósitos de pedido de patente, relacionados à determinada doença, poderia ser considerado uma medida confiável e válida para se aferirem os níveis de inovação farmacêutica na realidade brasileira.

Para tanto, realizou-se, na base de dados oficial do INPI, um levantamento do número pedidos de patente depositados, que fossem relacionados, diretamente, à doença de Chagas. E, em seguida, confrontaram-se os dados coletados com o conceito de inovação farmacêutica, de Marcia Angell, e de confiabilidade e validade das medidas, de Lee Epstein e Gary King.

Com base no aprendizado gerado pela observação desses dados empíricos (dados conhecidos), foi possível construir um processo inferencial descritivo, que resultou em novos dados sobre a realidade estudada (dados desconhecidos). Em primeiro lugar, foi possível inferir que o número de depósitos de pedidos de patente, relacionados à determinada doença, pode ser considerado uma medida confiável da inovação far- 
macêutica, caso a coleta dos depósitos, em base oficial do INPI, seja norteada por regras precisas, transparentes e replicáveis. Em segundo lugar, foi possível inferir que, apesar de confiável, por não ser considerada uma medida visual, imparcial e eficientemente válida, o número de depósitos de pedidos de patente, relacionados à determinada doença, não pode ser considerado uma medida válida para se aferirem os níveis de inovação farmacêutica na realidade brasileira.

Como parte de um diagnóstico empírico mais amplo, sobre as interações do sistema jurídico de patentes e o caso das doenças negligenciadas, essa investigação é apenas um primeiro estádio a ser consolidado. Tendo sido estabelecida a invalidade do número de pedidos de patente como medida da inovação farmacêutica, ainda neste momento de definição metodológica, serão realizadas análises qualitativas do conteúdo dos pedidos de patente concedidos pelo INPI, com o intuito de verificar a validade desse outro tipo de medida - amplamente utilizado pela literatura especializada na área.

Assim sendo, entende-se que, para se analisarem os impactos do atual modelo de incentivo à inovação na realidade dos países em desenvolvimento, é necessária uma abordagem metodológica criteriosa. Somente a partir de um diagnóstico confiável e válido da realidade, será possível fundamentar mudanças efetivas no sistema jurídico vigente e, consequentemente, criar condições melhores para todas as pessoas afetadas por doenças negligenciadas em todo o mundo.

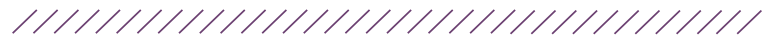

\section{Referências}

Angell, M. (2007). A verdade sobre os laboratórios farmacêuticos: como somos enganados e o que podemos fazer a respeito (5a ed.). (W. Barcellos, Trad.) Rio de Janeiro: Record. Obra original publicada em 2005).

Antunes, A. (Coord.). (2003). Subsídios a políticas públicas na área da saúde - inovação: Mapas de conhecimento sobre competências nacionais e tendências internacionais em doenças crônicas, doenças infecto-contagiosas e doenças negligenciadas. Brasília: CGEE. Recuperado em 14 setembro, 2017, de https://www.finep.gov.br/arquivos_ legados/fundos_setoriais/ct_saude/documentos/ ct-saude02mapa_conhecimentos_v3.pdf.

Boldrin, M., \& Levine, D. (2013). The Case Against Patents. Journal Of Economic Perspectives, 27(1), 3-22. http://dx.doi.org/10.1257/jep.27.1.3. Recuperado em 09 outubro, 2017, de https://www.aeaweb.org/articles?id=10.1257/jep.27.1.3.

Cruz, C. A. B. D., Silva, A. L. S., Alencar, E. M. D. D., Santos, N. D. J. B. D., Moreira, J. D. J. D. S., Paixão, A. E. A., \& Gomes, I. M. D. A. (2016). Tecnologias que empregam fármacos antiparasitários para tratamento da doença Chagas. RECIIS: Revista Eletrônica de Comunicação \& Informação \& Inovação em Saúde. (10)1, 1-9. Trimestral. Recuperado em 09 outubro, 2017, de https://www.reciis.icict.fiocruz.br/index. php/reciis/article/view/1075/pdf_1075>.

Drugs for neglected diseases initiative. (2017). Copa do Nordeste e Iniciativa Medicamentos para Doenças Negligenciadas se unem na luta contra a doença de Chagas. Recuperado em 09 outubro, 2017, de http://www.dndial.org/es/comunicacion-e-informacion/press-releases/2017/745-dndiecopadonordestecontrachagas.html.

Drugs for neglected diseases initiative. (2015). Relatório Anual: Medicamentos para populações negligenciadas. Rio de Janeiro: DNDi América Latina. Recuperado em 14 setembro, 2017, de http:// www.dndial.org/images/stories/pdf/docs/relatorioanual2015.

Epstein, L., \& King, G. (2013). Pesquisa Empírica em Direito: as regras de inferência. São Paulo: Direito GV. Recuperado em 21 outubro, 2016, de http://bibliotecadigital.fgv.br/dspace/handle/10438/11444. 
Feres, M. V. C., \& Silva, A. R. (2017). A aspiração do sistema de patentes e o caso dos produtos terapêuticos para doenças negligenciadas. REI - Revista Estudos Institucionais, 2(2), 756-798 (http:// dx.doi.org/10.21783/rei.v2i2.60). Recuperado em 09 outubro, 2017, de https://www.estudosinstitucionais.com/REl/article/view/60/115.

Garcia, D. L., \& Gonçalves, B. S. (2016). A influência dos investimentos públicos em CT\&l sobre o número de registros de patentes no estado de Sergipe no período de 2000 a 2013. 7th International Symposium on Technological Innovation 522-531 (http:// dx.doi.org/10.7198/s2318-3403201600030061.). Universidade Federal de Sergipe. Recuperado em 09 outubro, 2017, de http://www.api.org.br/ conferences/index.php/ISTI2016/ISTI2016/paper/ viewFile/35/88.

Gold, E. R., Kaplan, W., Orbinski, J., Harland-Logan, S., \& N-Marandi, S. (2010). Are Patents Impeding Medical Care and Innovation? Plos Medicine 7 (1), 1-5 (http://dx.doi.org/10.1371/journal. pmed.1000208. Public Library of Science (PLoS). Recuperado em 09 outubro, 2017, de http://journals.plos.org/plosmedicine/article?id=10.1371/ journal.pmed.1000208.

Hotez, P. J., \& Fujiwara, R. T. (2014). Brazil's neglected tropical diseases: An overview and a report card. Microbes And Infection 16, 601-606. Recuperado em 27 setembro, 2017, de http://www.ncbi.nlm. nih.gov/pubmed/25088506?dopt=Abstract.

Instituto Nacional da Propriedade Industrial (2017). Consulta à Base de Dados do INPI [base de dados eletrônica]. Recuperado em 07 julho, 2017, de https://gru.inpi.gov.br/pePI/servlet/ LoginController?action=login.

Resolução $n^{\circ} 80$ do Instituto Nacional da Propriedade Industrial de 19 de março de 2013 (2013). Disciplina a priorização do exame de pedidos de patente de produtos e processos farmacêuticos, bem como equipamentos e materiais relacionados à saúde pública. Recuperado em 27 setembro, 2017, de http:// www.inpi.gov.br/legislacao-arquivo/docs/resolucao_80-2013_-_exame_prioritario_saude.pdf.

Resolução $n^{\circ} 77$ do Instituto Nacional da Propriedade Industrial de 18 de março de 2013 (2013). Disciplina os procedimentos para a entrada na fase nacional dos pedidos internacionais de patente depositados nos termos do Tratado de Cooperação em Matéria de Patentes (PCT). Recuperado em 29 setembro, 2017, de http://www.inpi.gov.br/ legislacao-arquivo/docs/resolucao_77-2013.pdf.

Jannuzzi, A. H. L., \& Vasconcellos, A. G. (2017). Quanto custa o atraso na concessão de patentes de medicamentos para a saúde no Brasil? Cadernos de Saúde Pública 33(8) 1-6 (http://dx.doi. org/10.1590/0102-311x00206516.). FapUNIFESP (SciELO). Recuperado em 09 outubro, 2017, de http://www.scielo.br/pdf/csp/v33n8/1678-4464csp-33-08-e00206516.pdf.

Jannuzzi, A. H. L., \& Vasconcellos, A. G. (2013). Um estudo sobre a concessão de patentes de medicamentos no Brasil e suas implicações para a continuidade do êxito na política de medicamentos genéricos. ALTEC - XV Latino-iberoamericana de gestão de tecnologia. Porto. Recuperado em 23 agosto, 2017, em http://www.altec2013.org/programme_pdf/635.pdf.

Kesselheim, A. S, Wang, B, \& Avorn, J. D. (2013). "Innovativeness" in Drug Development: A Systematic Review. Clinical Pharmacology \& Therapeutics 94(3)336-348 (http://dx.doi.org/10.1038/ clpt.2013.115.). Springer Nature. Recuperado em 09 outubro, 2017, de http://onlinelibrary.wiley. com/doi/10.1038/clpt.2013.115/abstract.

Lei $n^{\circ}$ 9279, de 14 de maio de 1996 (1996). Regula direitos e obrigações relativos à propriedade industrial. Brasília, DF. Recuperado em 22 agosto, 2017, de http://www.planalto.gov.br/ccivil_03/leis/ L9279.htm.

Martins-Melo, F. R., Lima, M. D. S., Ramos Jr, A. N., Alencar, C. H., \& Heukelbach, J. (2014). Systematic review: Prevalence of Chagas disease in pregnant women and congenital transmission of Trypanosoma cruziin Brazil. Tropical Medicine \& International Health, 19 (8), 943-957 (http:// dx.doi.org/10.1111/tmi.12328.) Wiley-Blackwell. Recuperado em 09 outubro, 2017, de http://onlinelibrary.wiley.com/doi/10.1111/tmi.12328/abstr act;jsessionid=816872060E197EA58D307C7DAEC B9A31.f04t01.

Médecins Sans Frontières. (2016). Lives on the edge: time to align medical research and development with people's health needs (Relatório de Pesquisa/2016). Geneva: Médecins Sans Frontiè- 
res (MSF). Recuperado em 21 outubro, 2016, de http://www.msfaccess.org/content/report-lives-edge-time-align-medical-research-and-development-people's-health-needs.

Médicos Sem Fronteiras. (2013). Médica responde dúvidas sobre a doença de Chagas. Recuperado em 09 outubro, 2017, de http://www.msf.org.br/noticias/medica-responde-duvidas-sobre-doenca-de-chagas.

Pedrique, B., Strub-Wourgaft, N., Some, C., Olliaro, P., Trouiller, P., Ford, N., Pécoul, B., \& Bradol, J-H. (2013). The drug and vaccine landscape for neglected diseases (2000-11): a systematic assessment. The Lancet Global Health (1) 6. Recuperado em 09 outubro, 2017, de http://www.thelancet.com/journals/langlo/article/PIIS2214-109X(13)70078-0/ abstract>. Acesso em: 09 out. 2017.

Pires, Á. P. Sobre algumas questões epistemológicas de uma metodologia geral para as ciências sociais. In: POUPART, Jean et al. (2008). A Pesquisa Qualitativa: Enfoques epistemológicos e metodológicos 43-94. (Tradução de: A. C. A. Nasse, Trad). Petrópolis: Editora Vozes.

Pomin, F. M. D. C. (2015). Propriedade industrial como indicador de inovação na área farmacêutica: avaliação dos depósitos de patentes de laboratórios farmacêuticos brasileiros. Dissertação de Mestrado, Curso de Pós-graduação em Gestão, Pesquisa e Desenvolvimento na Indústria Farmacêutica, Instituto de Tecnologia em Fármacos Farmanguinhos, Rio de Janeiro, RJ, Brasil. Recuperado em 14 setembro, 2017, de https://www.arca.fiocruz. br/bitstream/icict/13003/1/10.pdf.

Silva, A. F. D. S. E, \& Luz Júnior, G. E. D. (2016). Prospecção tecnológica: potencialidades de diagnóstico para doenças de Chagas. Revista Brasileira de Inovação Tecnológica em Saúde (6), 2, 15-31, (http://dx.doi.org/10.18816/r-bits.v6i2.7400). Recuperado em 25 fevereiro, 2017, em https://periodicos.ufrn.br/reb/article/view/7400.

Tratado de Cooperação em Matéria de Patentes, de 9 de junho de 1970 (1970). Washington, DC. Recuperado em 09 outubro, 2017, de https://www.wipo. int/treaties/en/text.jsp?file_id=305907.

Data de submissão/Submission date: 08.04.2018.

Data de aceitação para publicação/Acceptance date: 06.12.2018. 